\title{
A kinematic examination of dual-route processing for action imitation
}

\author{
Arran T. Reader ${ }^{1,2}$ (D) Vaisnavi M. Rao ${ }^{2} \cdot$ Anastasia Christakou $^{2} \cdot$ Nicholas P. Holmes $^{3}$
}

Published online: 3 August 2018

(C) The Author(s) 2018

\begin{abstract}
The dual-route model of imitation suggests that meaningful and meaningless actions are processed through either an indirect or a direct route, respectively. Evidence indicates that the direct route is more cognitively demanding since it relies on mapping visuospatial properties of the observed action on to a performed one. These cognitive demands might negatively influence reaction time and accuracy for actions performed following a meaningless action under time constraints. However, how meaningful and meaningless action imitation processing is reflected in movement kinematics is not yet clear. We wanted to confirm whether meaningless action performance incurs a reaction time cost, whether the cost is reflected in kinematics, and, more generally, to examine kinematic markers of emblematic meaningful and meaningless action imitation. We examined participants' reaction time and wrist movements when they imitated emblematic meaningful or matched meaningless gestures in either blocks of the same action type or mixed blocks. Meaningless actions were associated with a greater correction period at the end of the movement, possibly reflecting a strategy designed to ensure accurate completion for less familiar actions under time constraints. Furthermore, in mixed blocks, trials following meaningless actions had a significantly increased reaction time, supporting previous claims that route selection for action imitation may be stimulus-driven. However, there was only convincing evidence for this effect with an interval of $\sim 2,948 \mathrm{~ms}$, but not $\sim 3,573 \mathrm{~ms}$ or $\sim 2,553 \mathrm{~ms}$, between movements. Future work motion-tracking the entire hand to assess imitation accuracy, and more closely examining the influence of duration between movements, may help to explain these effects.
\end{abstract}

Keywords Perception and action · Motor control · Neuropsychology

\section{Introduction}

Humans can copy actions that they are familiar with as well as new actions that they have never seen before. We can imitate actions that involve objects or have a goal, as well as emptyhanded actions that have no purpose whatsoever. Brain damage can negatively affect imitation. Apraxia, a disorder of complex

Electronic supplementary material The online version of this article (https://doi.org/10.3758/s13414-018-1582-z) contains supplementary material, which is available to authorized users.

Arran T. Reader

arran.reader@ki.se

1 Department of Neuroscience, Karolinska Institutet, Stockholm, Sweden

2 Centre for Integrative Neuroscience and Neurodynamics, School of Psychology and Clinical Language Sciences, University of Reading, Reading, UK

3 School of Psychology, University of Nottingham, Nottingham, UK movement, is one example where this can be the case. Patients with apraxia can show deficits in, among other skills, the ability to imitate meaningful or meaningless actions (Buxbaum \& Kalénine, 2010; Buxbaum \& Randerath, 2018; Canzano et al., 2016; Petreska et al., 2007; Rumiati et al., 2009).

The dissociation of deficits in meaningful and meaningless action imitation suggests a dual-route for different types of imitation, a model that has seen several developments and adaptations (see Buxbaum \& Randerath, 2018 or Petreska et al., 2007 for review). Broadly speaking, the dual-route approach to imitation suggests that meaningful actions are normally processed through a semantic, or indirect, route that draws upon stored pre-existing representations of those movements. By contrast, meaningless actions are processed using a direct route that relies on visuomotor mapping of the observed action in order to convert it into a performed action. Disruption to these routes could result in different performance deficits for different types of action imitation in apraxia (Tessari \& Cubelli, 2014).

What is less clear, however, is how these different routes manifest in healthy individuals. Whilst imitation has been 
much studied in healthy individuals, compared to work in apraxia less has been done to tease apart the ways in which meaningful and meaningless actions are imitated. Tessari and Rumiati (2004) examined this issue by testing healthy individuals' ability to rapidly imitate meaningful or meaningless actions in blocks of only one type of action, or in mixed blocks. Using a subjective rating measure, they found that performance for meaningful actions was significantly more accurate than meaningless actions when presented in blocks with only the same type of action, but that when meaningful and meaningless actions were presented together in mixed blocks, accuracy was not significantly different. They claimed that this supports a strategic selection of the dual-routes used for imitation: when participants could not be sure of the composition of the list of gestures to be imitated, they imitated solely using the direct route, bypassing semantic processing for meaningful actions and avoiding the cognitive cost of switching between the routes.

Press and Heyes (2008) replicated this experiment, and added a reaction time (RT) measure. Press and Heyes found that RT was shorter for meaningful actions in the blocked condition, but that this effect was not present in mixed conditions. To discover why this effect was not evident in the mixed blocks, they then looked at how imitation in the mixed blocks was affected by the action performed in the previous trial. Interestingly, they found that RTs were longer and movements less accurate when actions of either type followed a meaningless action in the mixed block. They suggested that this result supports a stimulus selection hypothesis: participants always used the processing route best suited to the action type, but the demands of the direct route (i.e., the cognitive demands of mapping the observed to a performed action) interferes with the speed of reaction to the following action. This reduces the advantage that meaningful actions have over meaningless actions.

In these previous experiments, the primary dependent variable was either RT or a subjective rating of accuracy. However, the spatial and kinematic elements of an observed action may be particularly useful for meaningless action imitation, and some apraxia research supports the idea that there may also be postural and kinematic dissociations in apraxic imitation deficits (Buxbaum et al., 2014; Dressing et al., 2016). Despite this, little research has been done to objectively characterize the kinematics of meaningful and meaningless action imitation in healthy individuals. Using motiontracking measures to examine how kinematics vary in different types of imitation may help develop our understanding of the behavioral differences in performing these types of action.

The aim of the experiments reported here was twofold. First, we wanted to further examine the dual-route processing of imitation, and to confirm the nature of route selection. We hypothesized that we would replicate the RT findings of Press and Heyes (2008), and find further support for the stimulus selection hypothesis. Secondly, we also wanted to study the broad-scale kinematic aspects of meaningless and meaningful action imitation with an exploratory examination of commonly used kinematic markers, with the hope that this would improve understanding of the underlying mechanisms of imitation of different action types. In particular, we were interested to find out if any kinematic markers would show similar effects to those observed in RT by Press and Heyes (2008).

\section{Experiment 1}

\section{Methods}

\section{Participants}

Twenty-four participants were recruited from the University of Reading and the surrounding area (mean $\pm \mathrm{SE}$ age $=24.4 \pm 1.08$ years, 11 males, three left-handed). Sample size was based on Press and Heyes (2008). The experimental procedures were approved by the local ethics committee (ref: 2016-059-AC). Participants gave written informed consent, and the experiments were conducted in accordance with the Declaration of Helsinki (as of 2008).

\section{Materials and stimuli}

The position of each participant's right wrist was recorded during action imitation using a wired Polhemus Fastrak (Polhemus Inc., Colchester, VT, USA) $120 \mathrm{~Hz}$ motiontracking system with six degrees of freedom (x, y, z, azimuth, elevation, and roll). The tracker was attached to the pisiform using adhesive medical tape and Velcro ${ }^{\mathrm{TM}}$.

The experiment was controlled and data were acquired using custom software written in MATLAB 2014a (Mathworks, Inc., Natick, MA, USA). We used the HandLabToolbox to control experiments and analyse data. The associated repositories are freely available at https:// github.com/TheHandLaboratory, whilst raw data are available on request.

A total of 20 intransitive gestures were used as stimuli. This included five meaningful emblematic hand gestures ("salute," "shock," "stop," "I'm listening," "looking into the distance"), five meaningful emblematic finger gestures ("peace," "thumbs up," "okay," "gun," "silence"), and ten matched meaningless gestures. For each meaningful gesture, a matched meaningless gesture was created (Fig. 1). In the case of finger gestures, this was done by changing the fingers used to create the gesture or the orientation of the hand. In the case of hand gestures, this was done by either changing the orientation or position of the hand. Intransitive emblematic gestures were used since these generally have a static endpoint that allows for the extraction of common kinematic markers (peak 
peace
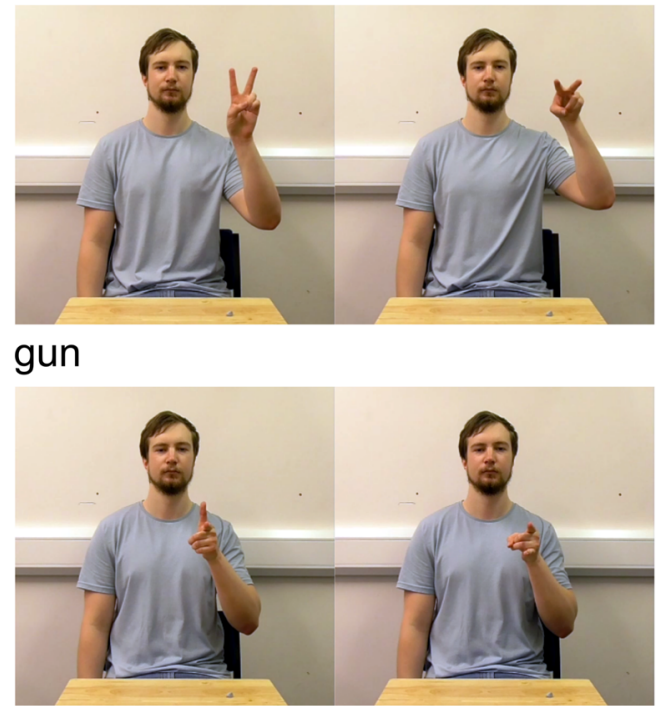

salute

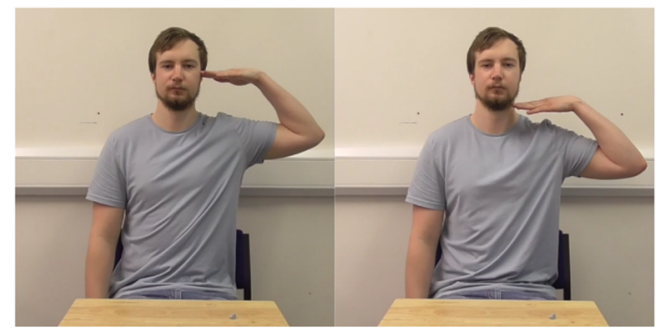

looking into the distance

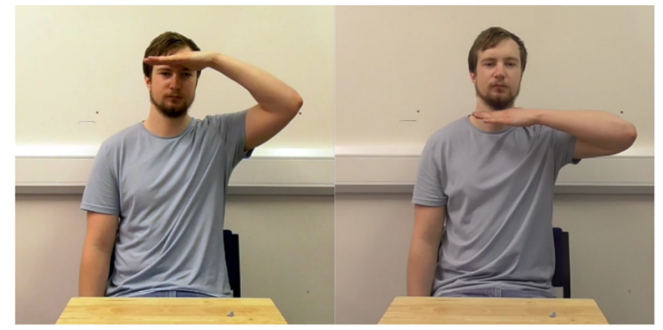

Fig. 1 Examples of gesture stimuli. For each meaningful emblematic gesture (left), a matched meaningless gesture was created (right)

acceleration, velocity, deceleration) from a bell-shaped velocity curve.

Gestures were presented as videos with a mean $\pm \mathrm{SD}$ duration of $1,573 \pm 16.1 \mathrm{~ms}$, in which a male actor raised his left hand from the table in front of him, created the gesture, and held it until the end of the video. The actor's gaze was fixed on the camera. Meaningful action videos had a mean \pm SD duration of $1,570 \pm 18.1 \mathrm{~ms}$, whilst meaningless action videos had a mean \pm SD duration of $1,576 \pm 14.3 \mathrm{~ms}$. In each video the mean \pm SD time to perform the initial action was $948 \pm 35.4 \mathrm{~ms}(943 \pm 32.8 \mathrm{~ms}$ for meaningful, $954 \pm 38.9$ for meaningless), after which the gesture was held in position until the end of the video. Videos were presented in the centre of a monitor at a resolution of $709 \times 591(18 \times 15 \mathrm{~cm}$ at 100 ppi) and $\sim 30$ frames/s.
During the task, participants sat opposite a $40 \mathrm{~cm}$ (diagonal measure) laptop computer screen approximately $110 \mathrm{~cm}$ away. A start point was placed on a table in front of the participant using Blu Tack ${ }^{\circledR}, 20 \mathrm{~cm}$ away from their right-hand side.

\section{Procedure}

Participants took part in four separate blocks of imitation, each with 80 trials. Two of the blocks contained only meaningful or meaningless gestures, each repeated eight times and pseudorandomly ordered (the blocked condition). The other two mixed blocks contained 50\% meaningful and 50\% meaningless gestures, with each gesture repeated four times and pseudorandomly ordered (the mixed condition). The order of these four blocks was counterbalanced across participants.

In a single trial, participants observed the action stimulus until the video finished. The screen then turned black, and a $100 \mathrm{~ms}$ tone signalled participants to repeat the action and maintain the gesture until they heard a further, lowerfrequency tone (lasting 250ms) 1,250ms later, at which point they were to return their hand to the start point. From the start of the second tone, participants had $750 \mathrm{~ms}$ to return their hand to the start point before the next trial began. Participants were asked to start the movement as quickly as possible after the cue and to imitate as accurately as possible. Once the four blocks were finished, participants completed a short questionnaire showing a pseudorandomized list of the gestures they had performed. For each gesture, they were asked to state whether they thought the action was meaningful, and if so what that action meaning was.

Trials were excluded from analysis if they contained a false start (wrist velocity $>10 \mathrm{~cm} / \mathrm{s}$ at the start of the trial), an unusually short movement duration $(\leq 200 \mathrm{~ms})$, or failure to finish the gesture before the end of the action period (wrist velocity did not drop below $10 \mathrm{~cm} / \mathrm{s}$ before the end of the trial). Participants were excluded if more than $25 \%$ of their trials in each block met the aforementioned trial exclusion criteria. Participants were also excluded if their subjective rating of the action meanings failed to meet an arbitrary $60 \%$ agreement with our own categorisation (mean $\pm \mathrm{SE}$ accuracy following exclusions $=82.1 \pm 2.03 \%$ ). These criteria resulted in one participant excluded due to their questionnaire results, and six due to an inadequate number of trials available for analysis. The excluded participants were replaced until a total of 24 participants were available for analysis.

\section{Data analysis}

For each participant, the data for meaningful and meaningless gestures in the mixed blocks were extracted and split into blocks of 80 meaningful and 80 meaningless trials. This meant that the four crossed conditions for the final analysis, with 80 
trials each, were blocked meaningful, blocked meaningless, mixed meaningful, and mixed meaningless.

An automated script was used for pre-processing and extraction of variables. The analysis routines processed the position data from each trial of each participant and rejected artefacts before filtering with a bidirectional low-pass fourthorder Butterworth filter (cut-off frequency $15 \mathrm{~Hz}$ ). Aside from RT, commonly used kinematic variables were extracted from the imitator's primary movements (i.e., from movement onset, when wrist velocity was greater than $5 \mathrm{~cm} / \mathrm{s}$ but not more than $10 \mathrm{~cm} / \mathrm{s}$, to the point at which the hand gesture was maintained and wrist velocity dropped below $10 \mathrm{~cm} / \mathrm{s}$ ) for exploratory analysis: movement time (MT, the duration of the wrist trajectory during the gesture in $\mathrm{ms}$ ), peak acceleration (PA, the maximum wrist acceleration), time to peak acceleration (TPA, the absolute time in ms to reach PA), TPA/MT (time to peak acceleration relative to the MT), peak velocity (PV, the maximum wrist velocity), time to peak velocity (TPV), TPV/ $\mathrm{MT}$, peak velocity/mean velocity (PV/MV, a measure of the narrowness of the velocity curve, i.e., kurtosis), peak deceleration (PD, the maximum wrist deceleration), time to peak deceleration (TPD), TPD/MT, and root mean squared jerk (RMS jerk, the third differential of the position data, a measure of wrist trajectory smoothness).

$11.3 \%$ of trials were removed due to false starts, an unusually short movement duration, or failure to finish the gesture before the end of the action period, and a further $1.7 \%$ of trials were removed due to remaining electromagnetic artefacts based on visual inspection. We decided post hoc to use the percentage of trials in which the actions were incomplete within the time limit as a further variable for analysis, to examine whether participants' ability to adequately complete the action was significantly reduced in any condition.

For each of the extracted variables, two-way repeated measures ANOVAs were run using JASP 0.8.6 (JASP Team, 2018) to compare the across-trial mean values per participant over the four conditions resulting from crossing block type and meaning. For RT we used another two-way repeated measures ANOVA to further examine whether the prior action (i.e., meaningful or meaningless) influenced the present action within the mixed block (as was found by Press \& Heyes, 2008). In these ANOVAs, prior and present action type were used as levels in a within-participant variable. We also performed this analysis on any exploratory variables in which we observed a statistically significant block type*meaning interaction.

Since our examination of RT was a confirmatory procedure based on the results of Press and Heyes (2008), we used a typical alpha of .05 for assessing statistical significance when analysing this variable. In order to control the false discovery rate (FDR) for the other (exploratory) kinematic variables, we used the correction procedure outlined by Benjamini and Yekutieli (2001) across all main effects and interactions for every exploratory variable assessed using the primary twoway ANOVA (i.e., creating a corrected alpha threshold for assessing statistical significance for all exploratory block type*meaning comparisons, and reporting only values equal to or less than this threshold as statistically significant).

Because this correction approach was particularly conservative, we also took steps to avoid type 2 errors - false negatives. We used a secondary, arbitrary alpha threshold of .01 to highlight any effects that did not reach statistical significance using the aforementioned correction procedure. To assess how likely any main effects that met this .01 alpha threshold would be under the null hypothesis, we performed two-sided Bayesian paired samples t-tests using JASP.

In the case of block type*meaning interactions meeting the .01 alpha threshold, we examined these in the same fashion, using the interaction term ((blocked meaningful - blocked meaningless) - (mixed meaningful - mixed meaningless)) for a one-sample comparison (test value $=0$ ). If there was positive evidence in support of $\mathrm{H} 1\left(\mathrm{BF}_{10}>3\right)$, we ran post hoc paired comparisons using Bonferroni-corrected two-tailed paired t-tests, two-sided Bayesian paired samples t-tests, and also assessed whether this effect could be explained by differences in prior action in the mixed blocks, using an ANOVA as described for RT above.

A default Cauchy prior of 0.707 , zero-centred, was used for all Bayesian analyses, unless otherwise stated. We used Raftery's (1995) criteria for interpreting the level of evidence for $\mathrm{H} 1$ or $\mathrm{H} 0$ provided by the Bayes factor. In support of $\mathrm{H} 1$, $\mathrm{BF}_{10}$ of $1-3$ is considered weak evidence, $\mathrm{BF}_{10}$ of $3-20$ is positive evidence, $\mathrm{BF}_{10}$ of $20-150$ is strong evidence, and $\mathrm{BF}_{10}>150$ is very strong evidence. The same numeric values of $\mathrm{BF}_{01}$ reflect the same degree of support for $\mathrm{H} 0$.

\section{Results}

\section{Reaction time}

We did not observe any significant difference in RT between meaningful (mean $\pm \mathrm{SE}=320 \pm 12.7 \mathrm{~ms}$ ) and meaningless $(312 \pm 10.2 \mathrm{~ms})$ actions $\left(\mathrm{F}(1,23)=1.67, \mathrm{p}=.209, \mathrm{\eta}_{\mathrm{p}}{ }^{2}=.068\right)$, or between blocked $(315 \pm 11.7 \mathrm{~ms})$ and mixed $(317 \pm 11.5 \mathrm{~ms})$ trials $\left(\mathrm{F}(1,23)=0.124, \mathrm{p}=.728, \mathrm{\eta}_{\mathrm{p}}{ }^{2}=.005\right)$. There was also no significant block type*meaning interaction $(\mathrm{F}(1,23)=1.00, \mathrm{p}=.328$, $\eta_{\mathrm{p}}{ }^{2}=.042$ ), suggesting that there was no effect of changing the block type on meaningful or meaningless action performance.

When examining trial-by-trial effects in the mixed blocks, there was no significant difference between prior meaningful $(316 \pm 11.4 \mathrm{~ms})$ and prior meaningless $(318 \pm 12.0 \mathrm{~ms})$ trials $\left(\mathrm{F}(1,23)=0.562, \mathrm{p}=.461, \mathrm{\eta}_{\mathrm{p}}{ }^{2}=.024\right)$, or between present meaningful $(318 \pm 12.2 \mathrm{~ms})$ and present meaningless $(316 \pm 11.2)$ trials $\left(\mathrm{F}(1,23)=0.560, \mathrm{p}=.462, \mathrm{n}_{\mathrm{p}}{ }^{2}=.024\right)$. There was no significant prior*present interaction $(\mathrm{F}(1,23)=0.007, \mathrm{p}=.934$, 
$\left.\eta_{\mathrm{p}}{ }^{2}<.001\right)$. These results were against what we had hypothesized, and what was shown for RT by Press and Heyes (2008).

\section{Exploratory kinematics}

When comparing kinematic variables (Table 1), we observed that meaningless trials had a significantly longer MT, and a significantly earlier TPA and TPV as a proportion of the MT, suggesting a prolongation of the velocity curve in the later phases of movement. There were no other statistically significant main effects or interactions using the FDR-corrected alpha threshold (Online Supplementary Table 1). However, when using an uncorrected arbitrary alpha threshold of .01, we observed that PV was greater for meaningless $(91.2 \pm 3.35 \mathrm{~cm} / \mathrm{s})$ compared to meaningful $(89.3 \pm 3.44 \mathrm{~cm} / \mathrm{s})$ trials $\left(\mathrm{F}(1,23)=8.76, \mathrm{p}=.007, \mathrm{\eta}_{\mathrm{p}}{ }^{2}=.276\right), \mathrm{BF}_{10}=6.53$. We also observed that TPD/MT was smaller for meaningless $((.756 \pm .00858)$ compared to meaningful $(.773 \pm .00894)$ trials $\left(\mathrm{F}(1,23)=9.58, \mathrm{p}=.005, \mathrm{\eta}_{\mathrm{p}}{ }^{2}=.294\right), \mathrm{BF}_{10}=8.56$.

\section{Discussion}

We found several interesting differences in kinematic parameters between performance for meaningful and meaningless actions. First, meaningless trials had a significantly greater MT and proportionally earlier TPA and TPV compared to meaningful actions. A Bayesian analysis of TPD/MT suggested that there was evidence for an effect in the same direction in this variable $\left(\mathrm{BF}_{10}>3\right)$. Such results indicate that the difference in MT reflects a difference in time between peak deceleration and the end of the action. It took longer for participants to settle into the final posture in meaningless actions, possibly because they were less familiar with the correct end point in these instances. The increased $\mathrm{PV}\left(\mathrm{BF}_{10}>3\right)$ observed in meaningless trials may also reflect this, such that participants performed less familiar actions more rapidly to be certain of their completion within the given time, and allowing themselves more time for correction. Overall, this finding seems to indicate that the correction period (encompassing the latter part of the deceleration phase) in gesture imitation is dependent on whether the imitator is familiar with the action.

Contrary to our hypothesis, and out of line with the research we were attempting to replicate, we did not observe a significant block type*meaning interaction in RT, or an influence of prior meaningless action in the mixed block. In this experiment, participants were required to watch the stimuli $(\sim 1,573 \mathrm{~ms})$, before they were given $1,250 \mathrm{~ms}$ to perform and maintain the gesture, then $750 \mathrm{~ms}$ to return their hand before the next trial started. This meant that the time between action onsets in consecutive trials (i.e., the trial length) was $\sim 3,573 \mathrm{~ms}$. This was more than the trial lengths in which (effects were found in the experiments of Tessari and Rumiati (2004) and Press and Heyes (2008): 1,750ms and $2,750 \mathrm{~ms}$, respectively.

As emphasized in the introduction, Press and Heyes (2008) suggested that, since the direct route for processing meaningless actions is more cognitively demanding, the increased working memory load associated with performing these sorts of actions can negatively influence the performance of the following action in the mixed block. We decided that participants may have had too much time to recover from the cognitive demands of the direct route following meaningless action imitation in the mixed blocks. This may have reduced the influence of prior meaningless action in the mixed blocks, thus reducing our likelihood of observing the block type*meaning interaction in RT discovered by Press and Heyes (2008). This may also explain why we did not observe any significant block type*meaning interactions in the other kinematic variables.

We attempted to rectify this in two further experiments, reducing the trial length, and therefore the interval between movement onsets, by increasing durations. We did this by manipulating our original stimuli of $\sim 1,573 \mathrm{~ms}$ duration. In Experiment 2 we examined $\sim 948 \mathrm{~ms}$ stimuli, showing the movement start to the gesture position being held. In Experiment 3 we examined $\sim 553 \mathrm{~ms}$ stimuli, simply showing the gesture position being held. We hypothesized that we would at least observe the RT interaction effect found in previous work, possibly in Experiment 2, but more likely in Experiment 3.

Table 1 Experiment 1 group means and statistically significant ANOVA results for the main effect of action meaning (FDR-corrected alpha threshold = .000281)

\begin{tabular}{|c|c|c|c|c|c|}
\hline \multirow[t]{2}{*}{ Variable } & \multicolumn{2}{|c|}{ Mean $( \pm \mathrm{SE})$ value } & \multicolumn{3}{|c|}{ Main effect of meaning } \\
\hline & Meaningful & Meaningless & $\mathrm{F}(1,23)$ & $\mathrm{p}$ & $\eta_{\mathrm{p}}^{2}$ \\
\hline TPA/MT (0-1) & $.188(.00809)$ & $.178(.00757)$ & 18.3 & .000281 & .443 \\
\hline TPV/MT (0-1) & $.467(.00852)$ & $.452(.00798)$ & 20.4 & .000157 & .469 \\
\hline MT (ms) & $659(11.9)$ & $684(11.2)$ & 44.4 & .000000846 & .659 \\
\hline
\end{tabular}




\section{Experiment 2}

\section{Methods}

The methods were the same as Experiment 1, except where indicated.

\section{Participants}

Twenty-four new participants were recruited (mean \pm SE age $=$ $20.2 \pm 0.54$ years, one male, one left-handed). None of the participants had taken part in the previous experiment. As in Experiment 1, participants were excluded if their subjective rating of the action meanings failed to meet a $60 \%$ agreement with our own categorisation (mean $\pm \mathrm{SE}$ accuracy following exclusions $=82.7 \pm 2.17 \%$ ). They were also excluded if every block had more than $25 \%$ of trials removed. Only the trialbased criteria resulted in exclusion (seven participants). These participants were replaced until 24 total participants were available for analysis.

\section{Materials, stimuli, and procedure}

These were the same as Experiment 1, except in this case the videos presented to participants had a mean \pm SD duration of $948 \pm 35.4 \mathrm{~ms}$. These videos were identical to those in Experiment 1, the only change being that they ended as soon as the posture was complete (i.e., excluding the period in which the gesture was held statically). Meaningful action videos had a mean \pm SD duration of $943 \pm 32.8 \mathrm{~ms}$, whilst meaningless action videos had a mean \pm SD duration of $954 \pm 38.9 \mathrm{~ms}$.

\section{Data analysis}

The analysis routine was as in Experiment 1. In this instance $4.44 \%$ of trials were removed due to false starts or failure to finish the gesture before the end of the action period, and a further $1.69 \%$ of trials were removed due to electromagnetic artefacts.

One participant showed unusually low RTs (below 150ms, dark green points in Fig. 2). However, since RT was cued in this experiment, with the videos finishing prior to the tone signalling movement, it is likely that participants were anticipating the action onset. In addition, since our RT measures are based on the movement of the wrist, rather than a button press or release, we found low RT values in general. Finally, this participant did not show outlying values on any other variable, suggesting that they were completing the task as expected.

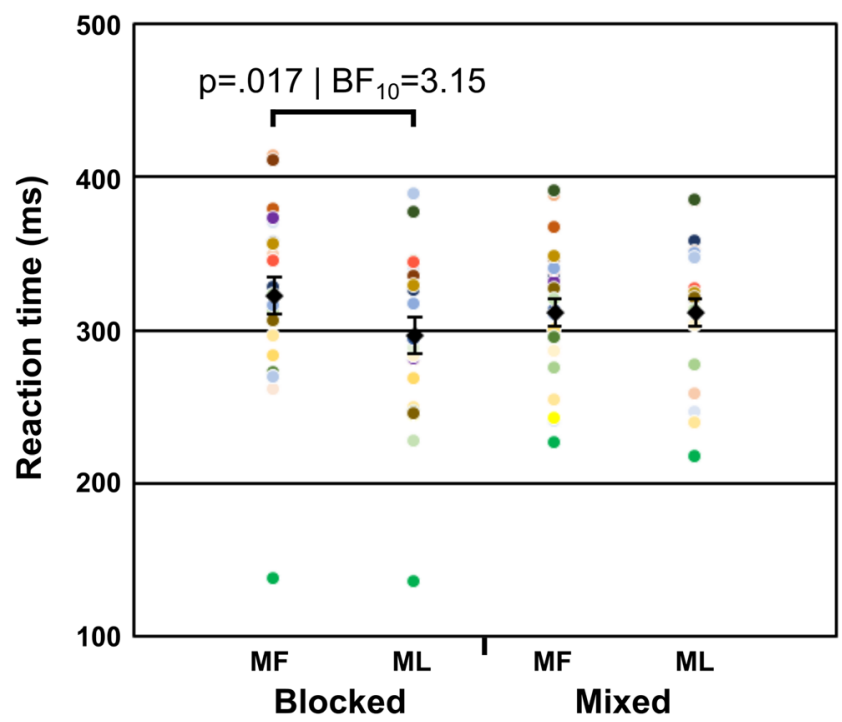

Fig. 2 Experiment 2 block type*meaning paired comparison for reaction time (RT). Colored points represent single participant values, black diamonds represent mean values with between-subjects SE bars. $M F$ meaningful, $M L$ meaningless

\section{Results}

\section{Reaction time}

In this experiment we observed an effect of action meaning on RT, such that meaningful actions (mean $\pm \mathrm{SE}=317 \pm 9.48 \mathrm{~ms}$ ) had a significantly longer RT than meaningless actions $\left(304 \pm 9.53 \mathrm{~ms}, \mathrm{~F}(1,23)=4.44, \mathrm{p}=.046, \mathrm{\eta}_{\mathrm{p}}{ }^{2}=.162\right)$. There was no significant difference between blocked $(309 \pm 10.7 \mathrm{~ms})$ and mixed $(311 \pm 8.97 \mathrm{~ms})$ trials $(\mathrm{F}(1,23)=0.061, \mathrm{p}=.808$, $\left.\eta_{\mathrm{p}}{ }^{2}=.003\right)$. There was a significant block type*meaning interaction $\left(\mathrm{F}(1,23)=9.52, \mathrm{p}=.005, \mathrm{\eta}_{\mathrm{p}}{ }^{2}=.293\right)$.

To examine this significant interaction (Fig. 2) we ran pairwise comparisons using two-tailed paired samples t-tests and two-sided paired samples Bayesian t-tests. Meaningful trials had a significantly longer RT than meaningless trials in the blocked condition $\left(\mathrm{t}(23)=2.58, \mathrm{p}=.017, \mathrm{~g}_{\mathrm{rm}}=0.431\right)$, $\mathrm{BF}_{10}=3.15$. However, there was no significant difference in RT between meaningful and meaningless trials in the mixed condition $\left(\mathrm{t}(23)=-0.0482, \mathrm{p}=.962, \mathrm{~g}_{\mathrm{rm}}=0.00322\right), \mathrm{BF}_{01}=4.65$.

Examining the mixed blocks revealed that in trials where the prior action was meaningless, RT was significantly greater than when the prior action was meaningful $(314 \pm 9.12 \mathrm{~ms}$ vs. $\left.307 \pm 9.22 \mathrm{~ms}, \mathrm{~F}(1,23)=5.97, \mathrm{p}=.023, \mathrm{\eta}_{\mathrm{p}}{ }^{2}=.206\right)$, as was observed by Press and Heyes (2008). There was no significant difference in RT between present meaningful $(311 \pm 9.11 \mathrm{~ms})$ and present meaningless $(310 \pm 9.20 \mathrm{~ms})$ actions $\left(\mathrm{F}(1,23)=0.019, \mathrm{p}=.893, \mathrm{\eta}_{\mathrm{p}}{ }^{2}=.001\right)$. There was also no significant prior*present interaction $(\mathrm{F}(1,23)=2.59, \mathrm{p}=.121$, $\left.\eta_{\mathrm{p}}{ }^{2}=.101\right)$. 


\section{Exploratory kinematics}

When examining the exploratory kinematic variables across both block types (Table 2) we found that, compared to meaningful trials, meaningless trials had a significantly larger PA and MT, and significantly smaller TPV/MT and TPD/MT. The effects of action meaning on MT, TPV/MT, and TPD/MT were replicated from Experiment 1. There were no other statistically significant main effects or interactions using the FDR-corrected alpha threshold (Online Supplementary Table 2).

However, using the uncorrected .01 alpha threshold, we did observe that PV was greater for meaningless $(77.6 \pm 1.97 \mathrm{~cm} / \mathrm{s})$ compared to meaningful $(75.3 \pm 1.92 \mathrm{~cm} / \mathrm{s})$ trials $\left(\mathrm{F}(1,23)=12.5, \mathrm{p}=.002, \mathrm{\eta}_{\mathrm{p}}{ }^{2}=.351\right), \mathrm{BF}_{10}=21.1$. We also observed a block type*meaning interaction in $\mathrm{PV}$ $\left(\mathrm{F}(1,23)=9.25, \mathrm{p}=.006, \mathrm{\eta}_{\mathrm{p}}^{2}=.287\right)$. Examining the interaction term (mean $\pm \mathrm{SE}=-3.25 \pm 1.07 \mathrm{~cm} / \mathrm{s})$ with a two-sided Bayesian one-sample t-test revealed $\mathrm{BF}_{10}=7.67$. In order to examine this interaction further, we ran pairwise comparisons using four two-tailed paired samples t-tests (Bonferronicorrected alpha .0125) and four two-sided paired samples Bayesian t-tests (Fig. 3A).

Meaningless actions in the blocked condition had a significantly greater PV than meaningful actions in the blocked condition $\left(\mathrm{t}(23)=3.78, \mathrm{p}<.001, \mathrm{~g}_{\mathrm{rm}}=0.351\right), \mathrm{BF}_{10}=36.1$. Meaningful actions in the blocked condition had a significantly smaller PV than meaningful actions in the mixed condition $\left(\mathrm{t}(23)=-2.86, \mathrm{p}=.009, \mathrm{~g}_{\mathrm{rm}}=0.327\right), \mathrm{BF}_{10}=5.33$. There was no significant difference in $\mathrm{PV}$ between meaningless actions in the blocked condition and meaningless actions in the mixed condition $\left(\mathrm{t}(23)=-0.053, \mathrm{p}=.958, \mathrm{~g}_{\mathrm{rm}}=0.00675\right), \mathrm{BF}_{01}=4.65$. There was also no significant difference in PV between meaningful actions in the mixed block and meaningless actions in the mixed block $\left(\mathrm{t}(23)=-1.19, \mathrm{p}=.248, \mathrm{~g}_{\mathrm{rm}}=0.0700\right)$, $\mathrm{BF}_{01}=2.49$. These results suggested that whilst PV for meaningful actions was lower than meaningless actions in the blocked trials, PV for meaningful actions increased to a similar level in the mixed condition. PV for meaningless actions remained static across blocked and mixed conditions.
Examining PV in the mixed blocks in terms of prior action type did not reveal an effect similar to that observed in RT. There was no significant difference in PV between prior meaningful $(76.8 \pm 1.92 \mathrm{~cm} / \mathrm{s})$ and prior meaningless $(77.5 \pm 1.91 \mathrm{~cm} / \mathrm{s})$ actions $\left(\mathrm{F}(1,23)=3.02, \mathrm{p}=.096, \mathrm{\eta}_{\mathrm{p}}{ }^{2}=.116\right)$, $\mathrm{BF}_{01}$ of 1.27. There was no significant difference in PV between present meaningful $(76.9 \pm 1.97 \mathrm{~cm} / \mathrm{s})$ and present meaningless $(77.5 \pm 1.87 \mathrm{~cm} / \mathrm{s})$ actions $(\mathrm{F}(1,23)=1.31, \mathrm{p}=.265$, $\left.\eta_{\mathrm{p}}{ }^{2}=.054\right)$. There was also no significant prior*present interaction $\left(\mathrm{F}(1,23)=3.32, \mathrm{p}=.082, \mathrm{\eta}_{\mathrm{p}}{ }^{2}=.126\right)$.

We also found that PV/MV was greater for meaningless (1.76 \pm 0.0111$)$ compared to meaningful $(1.74 \pm 0.0118)$ trials $\left(\mathrm{F}(1,23)=11.4, \mathrm{p}=.003, \mathrm{\eta}_{\mathrm{p}}{ }^{2}=.332\right), \mathrm{BF}_{10}=15.4$. We also observed a block type*meaning interaction in $\mathrm{PV} / \mathrm{MV}$ $\left(\mathrm{F}(1,23)=10.8, \mathrm{p}=.003, \mathrm{\eta}_{\mathrm{p}}^{2}=.320\right)$. When examining the interaction term (mean $\pm \mathrm{SE}=-0.0309 \pm 0.00939)$ with a two-sided Bayesian one-sample t-test we found $\mathrm{BF}_{10}=12.7$. To better understand this interaction, we ran pairwise comparisons using four two-tailed paired samples t-tests (Bonferronicorrected alpha .0125) and four two-sided paired samples Bayesian t-tests (Figure 3B).

Meaningless actions in the blocked condition had a significantly greater $\mathrm{PV} / \mathrm{MV}$ than meaningful actions in the blocked condition $\left(\mathrm{t}(23)=4.23, \mathrm{p}<.001, \mathrm{~g}_{\mathrm{rm}}=0.581\right)$, $\mathrm{BF}_{10}=97.4$. Meaningful actions in the blocked condition had a significantly smaller PV/MV than meaningful actions in the mixed condition $(\mathrm{t}(23)=-3.24, \mathrm{p}=.004$, $\left.\mathrm{g}_{\mathrm{rm}}=0.348\right), \mathrm{BF}_{10}=11.5$. There was no significant difference in PV/MV between meaningless actions in the blocked condition and meaningless actions in the mixed condition $\left(\mathrm{t}(23)=1.02, \mathrm{p}=.320, \mathrm{~g}_{\mathrm{rm}}=0.145\right), \mathrm{BF}_{01}=2.93$. There was also no significant difference in PV/MV between meaningful actions in the mixed block and meaningless actions in the mixed block $(\mathrm{t}(23)=-0.856, \mathrm{p}=.401$, $\left.\mathrm{g}_{\mathrm{rm}}=0.0960\right), \mathrm{BF}_{01}=3.35$. These results suggested that, similar to $\mathrm{PV}, \mathrm{PV} / \mathrm{MV}$ for meaningful actions was lower than for meaningless actions in the blocked trials, but increased to a similar level in the mixed condition. PV/MV for meaningless actions remained relatively static across blocked and mixed conditions.

Table 2 Experiment 2 group means and statistically significant ANOVA results for the main effect of action meaning (FDR-corrected alpha threshold = .000198)

\begin{tabular}{|c|c|c|c|c|c|}
\hline \multirow[t]{2}{*}{ Variable } & \multicolumn{2}{|c|}{ Mean $( \pm \mathrm{SE})$ value } & \multicolumn{3}{|c|}{ Main effect of meaning } \\
\hline & Meaningful & Meaningless & $\mathrm{F}(1,23)$ & $\mathrm{p}$ & $\eta_{\mathrm{p}}^{2}$ \\
\hline $\mathrm{PA}\left(\mathrm{cm} / \mathrm{s}^{2}\right)$ & $493(20.5)$ & $518(22.1)$ & 19.5 & .000198 & .459 \\
\hline TPV/MT (0-1) & $.481(.00756)$ & $.460(.00778)$ & 37.7 & .00000289 & .621 \\
\hline TPD/MT (0-1) & $.761(.00934)$ & $.734(.00853)$ & 34.8 & .00000515 & .602 \\
\hline MT (ms) & $622(10.4)$ & $648(11.4)$ & 20.3 & .000160 & .469 \\
\hline
\end{tabular}



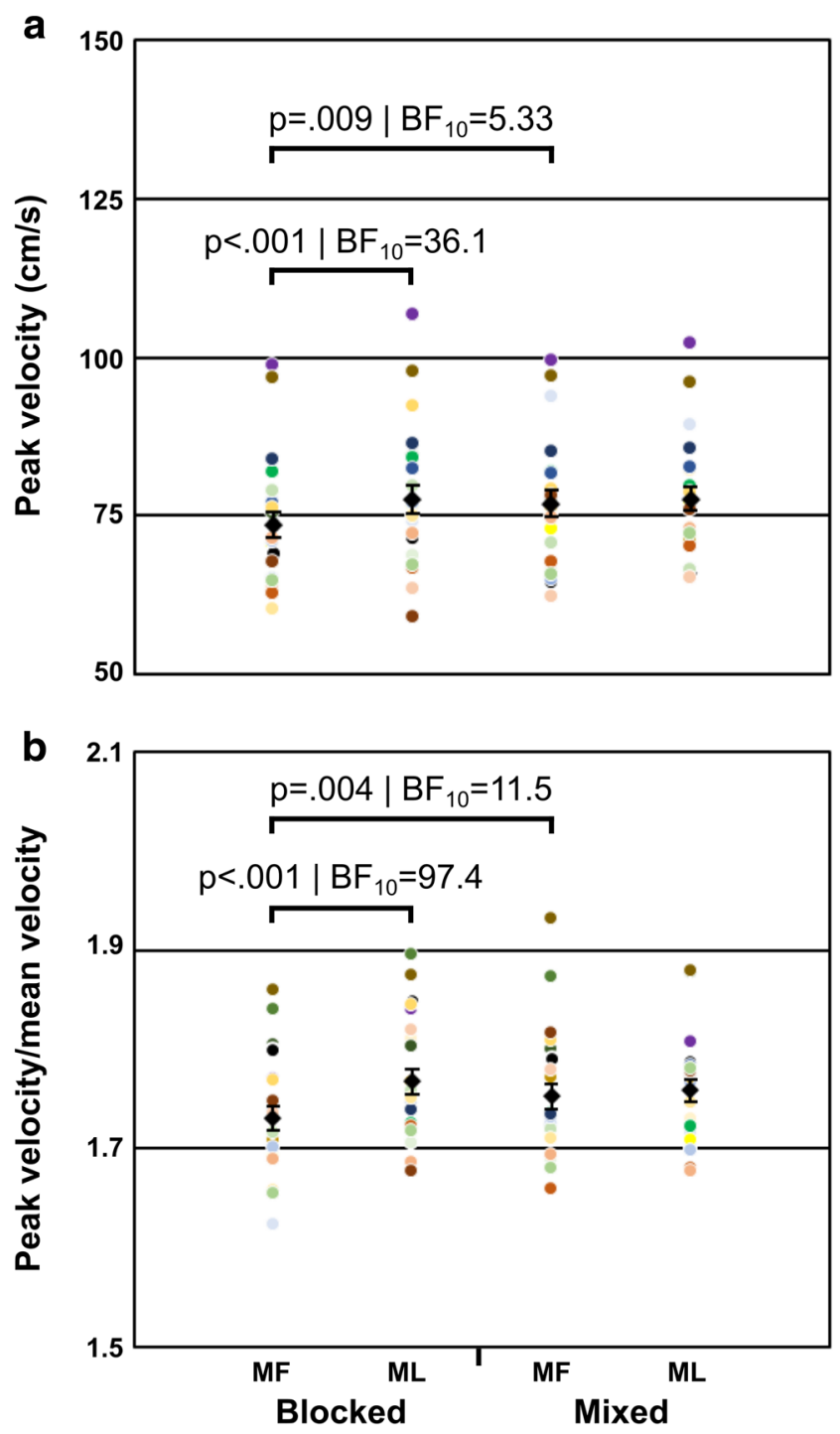

Fig. 3 Experiment 2 block type*meaning paired comparisons for (a) peak velocity and (b) peak velocity/mean velocity. Colored points represent single participant values, black diamonds represent mean values with between-subjects SE bars. $M F$ meaningful, $M L$ meaningless

Examining PV/MV in the mixed blocks in terms of prior action type did not result in a similar effect to that found for RT. There was no significant difference in PV/MV between prior meaningful $(1.76 \pm 0.0112)$ and prior meaningless $(1.75 \pm 0.0120)$ actions $\left(\mathrm{F}(1,23)=0.604, \mathrm{p}=.445, \mathrm{\eta}_{\mathrm{p}}{ }^{2}=.026\right)$, $\mathrm{BF}_{01}=3.55$. There was no significant difference in PV/MV between present meaningful $(1.75 \pm 0.0128)$ and present meaningless $(1.76 \pm 0.0107)$ actions $(\mathrm{F}(1,23)=0.616, \mathrm{p}=.440$, $\left.\eta_{\mathrm{p}}{ }^{2}=.026\right)$. There was also no significant prior*present interaction $\left(\mathrm{F}(1,23)=0.799, \mathrm{p}=.381, \mathrm{\eta}_{\mathrm{p}}{ }^{2}=.034\right)$.

\section{Discussion}

In this experiment RT was found to be significantly slower in imitation of meaningful compared to meaningless actions.
The block type*meaning interaction indicated that meaningful and meaningless actions were only significantly different in the blocked condition. When examining the mixed blocks, we observed that trials following a meaningless action were found to have a significantly greater RT compared to those following a meaningful action. This is in line with the findings of Press and Heyes (2008). This supports the idea that, in the mixed blocks, following the decreased interval between movement onsets in this experiment, the cognitive load associated with direct processing of meaningless gestures was so great as to interfere with the speed with which the following action was initiated, as suggested by Press and Heyes (2008).

Despite the replication of this effect, we observed that meaningful actions had a greater RT than meaningless actions in the blocked condition - the opposite direction of effect to that in Press and Heyes, (2008). This finding is probably due to differences between our experiment and that of Press and Heyes (2008), which are addressed in the general discussion below.

In keeping with Experiment 1, we also observed that meaningless actions had a significantly larger MT, and significantly smaller TPV/MT and TPD/MT than meaningful actions. This suggested that participants' behavior in terms of meaningful and meaningless action performance was broadly similar to that observed in Experiment 1. Participants used a greater correction time in meaningless actions, reflected by a longer period of movement following the time of PD. That meaningless actions had a significantly greater PA is again likely to reflect a more rapid performance of the action to allow for greater correction time in meaningless gestures (see Experiment 1, Discussion).

Interestingly, we observed significant block type*meaning interactions in PV and PV/MV. Paired comparisons suggested that $\mathrm{PV}$ and $\mathrm{PV} / \mathrm{MV}$ were greater for meaningless actions in the blocked condition - that is, meaningless actions were faster and had a more rapid transition between acceleration and deceleration. However, PV and PV/MV increased for meaningful actions in the mixed condition, to a similar level to that observed in meaningless actions. These variables did not significantly differ for meaningless actions across block types. Unlike RT, there was no significant effect of prior action in the mixed blocks for either of these variables. It is possible that these effects reflect a dual-route dissociation, though this may also be an influence of general motor-cognitive processing. For example, participants may have been more confident in their performance of meaningful actions in the blocked trials, such that they could afford a more leisurely approach to performing the action. The uncertainty regarding the block composition in the mixed trials may have resulted in a more rapid performance of known actions, perhaps due a perception of tighter time constraints.

Overall, the results of Experiment 2 support the results of Experiment 1 in indicating that when participants imitate 
meaningless actions they use a greater correction time after peak deceleration, and may speed the earlier stages of the action to provide for this. The results also seem to provide some support for the stimulus selection hypothesis posited by Press and Heyes (2008): imitating a meaningless action increases the RT of the following action, suggestive of a greater stress placed on cognitive resources by the direct route.

\section{Experiment 3}

In this experiment we further decreased the duration of gesture video stimuli, which we expected would increase the negative influence of meaningless action imitation on subsequent trial $\mathrm{RT}$ in the mixed blocks.

\section{Methods}

The methods were the same as for Experiment 2, except where indicated.

\section{Participants}

Twenty-four new participants were recruited (mean $\pm \mathrm{SE}$ age $=22.8 \pm 0.88$ years, seven male, three left-handed). None of the participants had taken part in the previous experiments. Participants' mean $\pm \mathrm{SE}$ agreement with our action categorisation was $78.3 \pm 1.75 \%$.

\section{Materials, stimuli, and procedure}

The videos presented to participants had a mean \pm SD length of $553 \pm 9.62 \mathrm{~ms}$. These videos were identical to those in the previous experiments, the only change being that they did not show the actor moving their hand to the gesture location, only holding the gesture in place for the full duration of the video. Meaningful action videos had a mean \pm SD duration of $551 \pm 13.6 \mathrm{~ms}$, whilst all meaningless action videos had duration of $555 \mathrm{~ms}$.

\section{Data analysis}

$9.41 \%$ of trials" were removed due to false starts, an unusually short movement duration, or failure to finish gesture before the end of the action period, and a further $2.07 \%$ of trials were removed due to electromagnetic artefacts.

\section{Results}

\section{Reaction time}

As in Experiment 2 we observed that meaningful actions (mean $\pm \mathrm{SE}=302 \pm 11.3 \mathrm{~ms}$ ) had a significantly longer RT than meaningless actions $(288 \pm 10.8 \mathrm{~ms}, \mathrm{~F}(1,23)=6.23, \mathrm{p}=.020$, $\left.\eta_{\mathrm{p}}{ }^{2}=.213\right)$. There was no significant difference in RT between blocked $(298 \pm 12.8 \mathrm{~ms})$ and mixed trials $(291 \pm 9.48 \mathrm{~ms}$, $\left.\mathrm{F}(1,23)=1.12, \mathrm{p}=.302, \mathrm{\eta}_{\mathrm{p}}{ }^{2}=.046\right)$, or any significant block type*meaning interaction $\left(\mathrm{F}(1,23)=0.042, \mathrm{p}=.840, \mathrm{\eta}_{\mathrm{p}}{ }^{2}=.002\right)$.

To understand why we no longer observed a block type*meaning interaction in this experiment, as we had observed in Experiment 2, we assessed the data using a Bayesian approach in JASP. We created difference values between the RTs for meaningful and meaningless trials in the blocked and mixed conditions (i.e., the interaction term) in Experiment 2. In order to create a posterior distribution of the interaction effect observed in Experiment 2, we performed a two-sided Bayesian one-sample t-test. This revealed a $\mathrm{BF}_{10}$ of 8.38 , suggesting positive evidence in favour of $\mathrm{H} 1$, for a difference between meaningful-meaningless RT difference values in blocked $(25.9 \pm 10.0 \mathrm{~ms})$ and mixed $(-0.149 \pm 3.08 \mathrm{~ms})$ trials (interaction term mean $\pm \mathrm{SE}=26.0 \pm 8.44 \mathrm{~ms}$ ) in Experiment 2 . The median effect size of the posterior distribution was 0.568 , lower $95 \%$ credible interval 0.149 , which is approximately comparable to a SD of 0.214 , assuming a normal distribution (assessed in $\mathrm{R}$ using the equation $(\operatorname{abs}(0.568-0.149) /$ qnorm $((1+0.95) / 2)))$.

We then used this posterior distribution as an informed normally distributed prior (half-normal centered on effect size $0.568, \mathrm{SD}=0.214$ ) in a one-sided Bayesian one-sample $\mathrm{t}$-test, to test the interaction values of Experiment 3 under the hypothesis that the difference in RT values between meaningful and meaningless actions in the blocked trials $(15.3 \pm 9.84 \mathrm{~ms})$ were greater than those in the mixed trials $(13.3 \pm 3.86 \mathrm{~ms})$, i.e., that the interaction term (mean $\pm \mathrm{SE}=$ $1.96 \pm 9.62 \mathrm{~ms}$ ) was greater than zero. We observed $\mathrm{BF}_{01}=7.03$. In the interest of comparison, we ran the same analysis on the Experiment 1 data, where we had also observed a non-significant interaction. The RT difference values in Experiment 1 were $14.3 \pm 12.1 \mathrm{~ms}$ in blocked trials, and $1.48 \pm 3.23 \mathrm{~ms}$ in mixed trials (interaction term mean $\pm \mathrm{SE}=12.9 \pm 12.9 \mathrm{~ms}$ ). This analysis revealed $\mathrm{BF}_{01}=1.84$.

When we ran a prior*present ANOVA on the Experiment 3 mixed block data to examine trial-by-trial effects, we observed that there was no significant difference in RT between prior meaningful $(288 \pm 10.3 \mathrm{~ms})$ or prior meaningless $(293 \pm 9.06 \mathrm{~ms})$ actions $\left(\mathrm{F}(1,23)=2.54, \mathrm{p}=.124, \mathrm{\eta}_{\mathrm{p}}{ }^{2}=.100\right)$, or significant prior*present interaction $\left(\mathrm{F}(1,23)=0.166, \mathrm{p}=.687, \mathrm{\eta}_{\mathrm{p}}{ }^{2}=.007\right)$. Importantly, we observed that present meaningful actions $(298 \pm 10.1 \mathrm{~ms})$ had a significantly greater RT than present meaningless actions $(284 \pm 9.50 \mathrm{~ms}, \mathrm{~F}(1,23)=13.1, \mathrm{p}=.001$, $\eta_{\mathrm{p}}{ }^{2}=.363$ ), reflecting the main effect of action meaning in the block type*meaning comparison above, which was not in keeping with our observations in Experiment 2 (where there was no significant difference in RT between meaningful and meaningless actions in the mixed block). 
To understand why we no longer observed an effect of prior meaningless action in the mixed block, as we had observed in Experiment 2, we performed a Bayesian analysis similar to the one reported above. In order to create a posterior distribution of the prior action effect observed in Experiment 2, we performed a two-sided Bayesian paired samples t-test. This analysis resulted in $\mathrm{BF}_{10}=2.46$. The median of the posterior distribution was 0.592 , with a lower $95 \%$ credible interval of 0.067 , approximately equivalent to an $\mathrm{SD}$ of 0.268 . We then used this posterior distribution as an informed normally distributed prior (half-normal centred on effect size $0.592, \mathrm{SD}=0.268$ ) in a one-sided Bayesian paired samples t-test, to test the prior action effect in Experiment 3 under the hypothesis that the RT values were greater when the prior action was meaningless compared to meaningful. We observed $\mathrm{BF}_{10}=1.58$. We ran the same analysis on the Experiment 1 data, where we had also observed a non-significant effect of prior action. The RT values in this instance were $316 \pm 11.4 \mathrm{~ms}$ in prior meaningful, and $318 \pm 12.0 \mathrm{~ms}$ in prior meaningless actions. This analysis revealed $\mathrm{BF}_{01}=2.90$. Neither of these effects provides evidence in either direction.

\section{Exploratory kinematics}

In the analysis of kinematic variables (Table 3) we observed that meaningless actions had a significantly greater TPD and MT, and significantly smaller TPV/MT and TPD/MT when compared to meaningful actions. These effects on MT, TPV/ MT, and TPD/MT were replicated from the previous experiments. There were no other statistically significant main effects or interactions (Online Supplementary Table 3).

Since we no longer observed significant block type*meaning interactions in $\mathrm{PV}$ and $\mathrm{PV} / \mathrm{MV}$, as in Experiment 2, we used the same Bayesian approach outlined above to use the posterior distributions of these previous interaction effects as informed priors. This was done in order to assess how likely our data was given $\mathrm{H} 0$ in these cases. Using a two-sided Bayesian one-sample t-test the posterior distribution of the block type*meaning interaction in $\mathrm{PV}$ in
Experiment 2 had a median of -0.558 and lower $95 \%$ credible interval of -1.00 , approximately equivalent to an SD of 0.226 . We used this posterior distribution as an informed normally distributed prior (half-normal centred on effect size -0.558 , $\mathrm{SD}=0.226)$ in a one-sided Bayesian one-sample t-test, to test the interaction values of Experiment 3 under the hypothesis that the difference in PV values between meaningful and meaningless actions in the blocked trials $(-1.53 \pm 1.54 \mathrm{~cm} / \mathrm{s})$ were greater in magnitude than those in the mixed trials $(0.207 \pm 0.508 \mathrm{~cm} / \mathrm{s})$ i.e., that the interaction term (mean \pm $\mathrm{SE}=-1.74 \pm 1.53 \mathrm{~cm} / \mathrm{s}$ ) was less than zero. We observed $\mathrm{BF}_{01}=1.35$. An analysis of the non-significant interaction in Experiment 1 (blocked difference $=-3.11 \pm 1.59 \mathrm{~cm} / \mathrm{s}$, mixed difference $=-0.761 \pm .499 \mathrm{~cm} / \mathrm{s}$, interaction term $=$ $-2.35 \pm 1.96 \mathrm{~cm} / \mathrm{s}$ ) resulted in $\mathrm{BF}_{01}=1.21$.

The posterior distribution of the block type*meaning interaction in PV/MV in Experiment 2 had a median of -0.611 and lower $95 \%$ credible interval of -1.06 , approximately equivalent to an SD of 0.229. We used this posterior distribution (created with a two-sided Bayesian one-sample t-test) as an informed normally distributed prior (half-normal centred on effect size $-0.661, \mathrm{SD}=0.229$ ) in a one-sided Bayesian onesample t-test, to test the interaction values of Experiment 3 under the hypothesis that the difference in PV/MV values between meaningful and meaningless actions in the blocked trials $(-0.0148 \pm 0.0101)$ were greater than those in the mixed trials $(-0.0108 \pm 0.00642)$, i.e., that the interaction term (mean $\pm \mathrm{SE}=-0.00399 \pm 0.0108$ ) was less than zero. We observed $\mathrm{BF}_{01}=8.68$. An analysis of the nonsignificant interaction in Experiment 1 (blocked difference $=$ $-0.0130 \pm 0.00854$, mixed difference $=-0.00915 \pm 0.00635$, in teraction term $=-0.00386 \pm 0.0104)$ resulted in $\mathrm{BF}_{01}=8.65$.

\section{Discussion}

In Experiment 3 we continued to find a significantly smaller TPV/MT and TPD/MT, and a greater MT for meaningless actions. We also observed that meaningless actions had a significantly later TPD, despite the earlier TPD/MT. The later

Table 3 Experiment 3 group means and statistically significant ANOVA results for the main effect of action meaning (FDR-corrected alpha threshold = .000954)

\begin{tabular}{|c|c|c|c|c|c|}
\hline \multirow[t]{2}{*}{ Variable } & \multicolumn{2}{|c|}{ Mean $( \pm \mathrm{SE})$ value } & \multicolumn{3}{|c|}{ Main effect of meaning } \\
\hline & Meaningful & Meaningless & $\mathrm{F}(1,23)$ & $\mathrm{p}$ & $\eta_{\mathrm{p}}^{2}$ \\
\hline TPV/MT (0-1) & $.479(.00948)$ & $.456(.0100)$ & 43.4 & .00000185 & .653 \\
\hline TPD (ms) & $476(12.2)$ & $487(12.6)$ & 14.3 & .000954 & .384 \\
\hline TPD/MT (0-1) & $.754(.00984)$ & $.726(.00979)$ & 34.3 & .00000578 & .598 \\
\hline MT (ms) & $631(11.1)$ & $673(12.1)$ & 107 & .000000000405 & .823 \\
\hline
\end{tabular}


TPD might reflect an attempt by participants to reduce the correction time in the face of a shorter trial duration (despite the time available for performing actions remaining the same). Since the trials were shorter, this may have biased participant behavior such that those in Experiment 3 felt a greater need to complete the actions more rapidly. However, since MT continued to be longer in meaningless actions, it is possible that this did not assist in reducing the correction time.

Whilst we found that meaningless actions had a significantly smaller RT compared to meaningful actions, this effect was not confined to the blocked trials, and there was no longer a significant block type*meaning interaction. Using a prior distribution informed by the interaction effect observed in Experiment 2 suggested positive evidence in favour of the null hypothesis $\left(\mathrm{BF}_{01}>3\right)$. We also failed to observe a statistically significant effect of prior meaningless action in the mixed blocks, though using a Bayesian analysis with a prior distribution informed by the effect observed in Experiment 2 provided us with weak evidence in favour of a similar effect. In addition, we failed to observe significant block type*meaning interactions in PV and PV/MV, with a Bayesian analysis suggesting weak evidence for the null hypothesis in the case of $\mathrm{PV}$, and positive evidence for the null hypothesis in the case of $\mathrm{PV} / \mathrm{MV}$.

\section{General discussion}

We used motion-tracking to examine the dual-route model of imitation in healthy participants. We hoped to reveal information about the kinematic differences between the imitation of meaningful and meaningless emblematic gestures, and attempted to replicate previous findings in RT from a previous experiment (Press \& Heyes, 2008). In terms of the wrist movements' kinematics, we found that meaningless action imitation was associated with a longer time spent in the "correction" phase, at the end of the movement, as compared to meaningful actions, and that participants tended to increase their movement speed when performing meaningless actions, perhaps in order to account for this.

When the previously observed RT effect (Press \& Heyes, 2008) was not evident in our original experiment (with stimuli of $\sim 1,573 \mathrm{~ms}$ in length, and $\sim 3,573 \mathrm{~ms}$ between movement onsets), we hypothesized that reducing the duration of stimuli (and therefore the total trial duration, and effectively the interval between movement onsets) could place greater stress on the direct route for matching observed and performed actions. We observed a similar effect to that revealed by Press and Heyes (2008) when we used a $248 \mathrm{~ms}$ stimulus duration (with $\sim 2948$ ms between movement onsets). Performance of a meaningless action in the mixed block resulted in a slower RT in the subsequent trial.

\section{Reaction-time effects}

\section{Influence of prior action in mixed blocks}

In Experiment 2 we observed that movements following a meaningless trial in the mixed blocks had a significantly reduced RT compared to movements following a meaningful trial. A similar significant effect was not observed in Experiment 1, where a Bayesian analysis instead revealed weak evidence in support of the null hypothesis.

Our results go some way to support the stimulus selection hypothesis posited by Press and Heyes (2008), which suggests that in mixed blocks actions are always imitated by way of their associated route when there is a time constraint. After reducing the time between movement onsets from that used in Experiment 1, participants in Experiment 2 were significantly slower to initiate both meaningful and meaningless actions following a meaningless trial in the mixed blocks. This could be suggestive of a lingering influence of increased cognitive load following direct processing of meaningless actions. The fact that this was only observed after meaningless actions makes it likely that only the direct route was used in these instances, for the purpose of matching an observed to a performed action (but see Rumiati \& Tessari, 2002; Tessari et al., 2006, 2007, 2009; Tessari \& Cubelli, 2014; Tessari \& Rumiati, 2004). Reducing the duration between movement onsets by $\sim 625 \mathrm{~ms}$ was enough to create this interference effect, and one possible explanation for the absent effect in Experiment 1 is that the interval between movement onsets was just beyond the limit in which we could reliably observe an influence of dual-route processing on RT.

What is less clear is why reducing the time available between movements by a further $\sim 395 \mathrm{~ms}$ would then negate this significant effect. A Bayesian analysis based on the posterior distribution of the effect of prior action observed in Experiment 2 suggested that there was only weak evidence for a similar effect in Experiment 3. It is possible that, since the semantic route could be the dominant route for imitation (Tessari et al., 2009), under such intense time constraints some participants reduced the accuracy of the meaningless (but not meaningful) actions in order to ensure task completion. This could in turn reduce the cognitive interference related to meaningless action performance in the mixed trials - fewer cognitive resources would be dedicated to accurate visuomotor matching of the observed meaningless action, meaning less negative influence on the following trial. Unfortunately, we did not record participant accuracy, but certainly this would provide a feasible explanation.

\section{Block type*meaning interactions}

We were surprised to observe in Experiment 2 that meaningful actions were performed with a slower RT than 
meaningless actions in the blocked trials, contrary to Press and Heyes (2008). A Bayesian analysis of this interaction revealed positive evidence in favor of the null hypothesis in Experiment 3. In Experiment 3 we instead observed a significant main effect, indicating that meaningful actions had a slower RT than meaningless actions in general. This was also reflected in a main effect of "present" actions in the mixed blocked ANOVA.

The significant block type*meaning interaction in Experiment 2 is not easily explained by the role of prior meaningless action in the mixed blocks. Press and Heyes (2008) observed that meaningful actions had a significantly faster RT than meaningless actions in the blocked trials, but not in mixed trials. Whilst the results of Press and Heyes (2008) indicate that the benefit of meaningful action performance is reduced in the mixed block (i.e., the RT increases), due to the interference of prior meaningless actions, our results are not in keeping with this observation (Fig. 2). In our case the block type*meaning interaction does not seem to reflect the influence of prior meaningless action in the mixed block. It is not clear what could be driving this inconsistent finding. It may be due to differences between our experiment and the one reported by Press and Heyes (2008).

In their experiment participants were required to imitate the action as quickly as possible from the video onset, unlike in our experiment in which participants had to first observe the action. In the experiment by Press and Heyes (2008), it is likely that meaningful action performance was speeded due to more rapid visual processing of familiar information. It may be this rapid visual processing that was influenced by the cognitive demands of direct route processing following prior meaningless actions in the mixed block. Generally speaking, visual processing could be less influential in our experiment, since participants had to wait until the end of the stimulus to begin to move (similar to some assessments for apraxia, e.g., Goldenberg, 1996). One consequence of this for meaningful and meaningless action performance is that meaningful actions could afford a slower RT, since the time required to perform a familiar action is presumably known in advance. This would only be true when participants are afforded the entire action prior to their movement, as in our experiments.

However, this reasoning does not seem to sufficiently explain the variability in block type*meaning interactions across experiments. It could be the case that, as described for the prior action effect above, participants implemented a speed-accuracy trade-off for meaningless actions in the mixed blocks in Experiment 3, but not Experiment 2, due to the increased time constraints. It appears that more work is needed to understand the relative roles of visual and cognitive processing in meaningful and meaningless action imitation, and how they might interact with task time constraints.

\section{Kinematics in meaningful and meaningless action imitation}

\section{Correction time as a marker for meaningless action performance}

Interestingly, we failed to observe effects of prior action in the mixed block in our exploratory kinematic parameters. One possibility is that these effects are simply not observable at the level of the wrist - they may be specific to the more finegrained contributors to the action (i.e., the fingers). The reported results for gesture accuracy by Tessari and Rumiati (2004) and Press and Heyes (2008) support this. However, in future work it may be beneficial to more closely examine the kinematics of the fingers to provide a more objective measure of imitation accuracy (e.g., Reader et al., 2018).

The most consistent exploratory kinematic effect, observed in all experiments (statistically significant at FDR-corrected threshold, or $\mathrm{BF}_{10}>3$ ), was that meaningless actions were associated with a longer MT, and proportionally earlier TPV and TPD, strongly indicative of a longer time spent in the final, corrective phase of the movement, as compared to meaningful actions. One possible explanation for this is that participants purposely maintained a greater correction time in actions they did not know, to give themselves more time to confirm that the final posture was accurate. The increased PV or PA for meaningless actions in Experiments 1 and 2 may also support this, and could reflect a strategy designed to increase movement speed in the early stages of a meaningless action in order to ensure adequate time for correction.

These results provide an interesting addition to our understanding of imitation. Whilst previous work has quantitatively examined the kinematic elements of imitative behavior in both healthy people (e.g., Braadbaart et al., 2012; Campione \& Gentilucci, 2011; Era et al., 2018; Forbes \& Hamilton, 2017; Gold et al., 2008; Hayes et al., 2016; Krüger et al., 2014; Pan \& Hamilton, 2015; Reader \& Holmes, 2015; Reader et al., 2018; Sacheli et al., 2012; Sacheli et al., 2013, Sacheli, Christensen, et al., 2015, Sacheli, Candidi, et al., 2015; Wild et al., 2010; Williams et al., 2013) and brain-damaged patients (e.g., Candidi et al., 2018; Hermsdörfer et al., 1996), as far as we are aware no previous experiments have looked at so many components of the velocity profile in order to compare the coarse-grained (i.e., wrist) kinematic approach to meaningful and meaningless action imitation. Much informative work has been done to assess action performance in meaningful and meaningless action imitation (e.g., Buxbaum et al., 2014; Carmo \& Rumiati, 2009; Goldenberg \& Hagmann, 1997; Mengotti et al., 2013), but frequently using only subjective rating measures.

It is essential to expand on our findings with further motion-tracking experiments, particularly if we want to understand how emblematic gesture imitation differs from more 
commonly examined point-to-point movements (i.e., reaching-to-grasp), or the object-directed action pantomimes frequently assessed in apraxia research. In addition, given the potential interactions between kinematic and semantic processing revealed by apraxia (Buxbaum et al., 2014; Dressing et al., 2016), further work using motion-tracking could be useful for understanding the neural control of imitation of different action types, particularly if combined with neuropsychological assessments or neuroimaging.

\section{Variability between experiment results}

It is worth briefly considering some of the more inconsistent kinematic effects between the three experiments reported here. Only in Experiment 1 did we observe that TPA/MT was significantly smaller for meaningless compared to meaningful actions, and only in Experiment 2 did we observe that PA was significantly greater for meaningless actions compared to meaningful actions. These results are broadly in keeping with the aforementioned correction time strategy - an increased PA and relatively earlier TPA could also contribute to a strategy aimed at a longer correction period. The variability between experiments on these parameters may be reflective of individual differences in speeding performance for meaningless actions: whilst some participants performed meaningless actions more rapidly by accelerating faster, some may have increased their speed (velocity) in general. This hypothesis would need further testing.

In Experiment 2 we also observed significant block type*meaning interactions in PV and PV/MV, suggesting that meaningless actions were faster, and with more rapid transition between acceleration and deceleration than meaningful actions only in the blocked condition. These variables increased in meaningful actions to match the values of the meaningless actions in the mixed blocks. There was weak (PV) and positive (PV/MV) evidence in favour of the null hypothesis when we examined this interaction effect in Experiments 1 and 3. These variables did not show significant effects of prior action type in the mixed block.

As discussed earlier, it is unclear whether these effects reflect dual-route processing, and they may instead reflect general motor-cognitive processes underlying performance under time constraints. Like the block type*meaning interaction in RT, one possibility is that participants could afford a slower speed and transition between acceleration and deceleration for meaningful actions when they were confident of the contents of the block. In the mixed block, which is perhaps more difficult in general, participants performed meaningful actions as rapidly as they would perform the novel meaningless actions, in order to optimize performance in a potentially more demanding task.

We suggest that in these cases it is possible that participants could, if they wished, perform the meaningful actions at a more leisurely pace than was observed. A comparison between predictable and unpredictable switches between action types in the mixed block (e.g., Tessari et al., 2009) would be useful for assessing this claim in future experiments. However, it is not clear why this effect was not similarly observed in Experiments 1 and 3. Again, further experiments would be necessary to better understand how the interval between action onsets might mediate these effects, which could feasibly be controlled by top-down cognition (e.g., Hamilton 2014, 2015).

Lastly, only in Experiment 3 did we observe that TPD was significantly later in meaningless as compared to meaningful trials. As highlighted in the discussion of Experiment 3, this may reflect an attempt to reduce the correction time under greater time pressure, rather than increasing velocity or acceleration at the start of the action.

\section{Limitations and future research}

One underlying problem with the experiments reported here is the variable proportion of movement shown in the different stimuli in each experiment. Based on previous evidence, it seems most likely that the reduction in stimulus duration was responsible for our RT results across experiments, and perhaps also the block type*meaning interaction observed in $\mathrm{PV}$ and $\mathrm{PV} / \mathrm{MV}$. However, it is still possible that the proportion of movement shown in the experimental stimuli could be involved in mediating responses in this task. Testing the role of observed movement to gesture may be a worthy avenue for future research.

Similarly, future work may also choose to examine further how imitation accuracy, rather than reaction time, is associated with dual-route processing of imitation under varying intermovement intervals. In addition, it would be interesting to know to what degree the correction time markers associated with novel action imitation are associated with visual or proprioceptive correction, especially since visual feedback has previously been found to be particularly useful for finetuning the endpoint of novel actions (Franklin et al., 2007).

\section{Conclusions}

We found that the time spent in the terminal, "correction" phase of movement is a distinguishing factor between the imitation of meaningful and meaningless actions. This effect was found in each of the three experiments reported here. In addition, our results broadly support a stimulus selection hypothesis for dual-route processing of imitation, though most convincingly under inter-movement intervals of $\sim 2,948 \mathrm{~ms}$. The effect was evident for reaction time measures, but not wrist kinematics. Further work should aim to better quantify the influence of the interval between movements on RT, and disentangle the interaction between kinematic parameters and 
accuracy in the dual-route processing of meaningful and meaningless action imitation. More comprehensive motiontracking of the arm and hand is likely to help us better understand the imitation of different types of action in healthy individuals, as well as imitation deficits in apraxia.

Acknowledgements Many thanks to Dr. Peter Scarfe for his help with coding the experiment and advice on an earlier version of this manuscript. This work was supported by the Economic and Social Research Council (grant number ES/J500148/1 to ATR), and the University of Reading Undergraduate Research Opportunities Program (to VMR).

Open Access This article is distributed under the terms of the Creative Commons Attribution 4.0 International License (http:// creativecommons.org/licenses/by/4.0/), which permits unrestricted use, distribution, and reproduction in any medium, provided you give appropriate credit to the original author(s) and the source, provide a link to the Creative Commons license, and indicate if changes were made.

\section{References}

Benjamini, Y., \& Yekutieli, D. (2001). The control of the false discovery rate in multiple testing under dependency. Annals of Statistics 29(4), $1165-1188$.

Braadbaart, L., Waiter, G.D., \& Williams, J.H.G. (2012). Neural correlates of individual differences in manual imitation fidelity. Frontiers in Integrative Neuroscience, 6, 91. https://doi.org/10.3389/fnint. 2012.00091

Buxbaum, L.J., \& Kalénine, S. (2010). Action knowledge, visuomotor activation, and embodiment in the two action systems. Annals of the New York Academy of Sciences, 1191, 201-218. https://doi.org/10. $1111 / \mathrm{j} .1749-6632.2010 .05447 . \mathrm{x}$

Buxbaum, L.J., \& Randerath, J. (2018). Limb apraxia and the left parietal lobe. Handbook of Clinical Neurology, 151, 349-363. https://doi. org/10.1016/B978-0-444-63622-5.00017-6

Buxbaum, L.J., Shapiro, A.D., \& Coslett, H.B. (2014). Critical brain regions for tool-related and imitative actions: A componential analysis. Brain, 137, 1971-1985. https://doi.org/10.1093/brain/awu111

Campione, G.C., \& Gentilucci, M. (2011). Is the observation of the human kinematics sufficient to activate automatic imitation of transitive actions? Behavioural Brain Research, 225, 201-208. https:// doi.org/10.1016/j.bbr.2011.07.025

Candidi, M., Sacheli, L.M., Era, V., Canzano, L., Tieri, G., \& Aglioti, S.M. (2018). Come together: Human-avatar on-line interactions boost joint-action performance in apraxic patients. Social Cognitive and Affective Neuroscience, 12(11), 1793-1802. https:// doi.org/10.1093/scan/nsx114

Canzano, L., Scandola, M., Gobbetto, V., Moretto, G., D'Imperio, D., \& Moro, V. (2016). The representation of objects in apraxia: From action execution to error awareness. Frontiers in Human Neuroscience, 10(39). https://doi.org/10.3389/fnhum.2016.00039

Carmo, J.C., \& Rumiati, R.I. (2009). Imitation of transitive and intransitive actions in healthy individuals. Brain and Cognition, 69, 460464. https://doi.org/10.1016/j.bandc.2008.09.007

Dressing, A., Nitschke, K., Kümmerer, D., Bormann, T., Beume, L., Schmidt, C.S.M., Ludwig, V.M., Mader, I., Willmes, K., Rijntjes, M., Kaller, C.P., Weiller, C., \& Martin, M. (2016). Distinct contributions of dorsal and ventral streams to imitation of tool-use and communicative gestures. Cerebral Cortex, 28(2), 474-492. https:// doi.org/10.1093/cercor/bhw383
Era, V., Candidi, M., Gandolfo, M., Sacheli, L.M., \& Aglioti, S.M. (2018). Inhibition of left anterior intraparietal sulcus shows that mutual adjustment marks dyadic joint-actions in humans. Social Cognitive and Affective Neuroscience, 13(5), 492-500. https://doi. org/10.1093/scan/nsy022

Forbes, P.A.G., \& Hamilton, A.F. de C. (2017). Moving higher and higher: Imitators' movements are sensitive to observed trajectories regardless of action rationality. Experimental Brain Research, 235(9), 2741-2753. https://doi.org/10.1007/s00221-017-5006-4

Franklin, D.W., So, U., Burdet, E., \& Kawato, M. (2007). Visual feedback is not necessary for the learning of novel dynamics. PLoS ONE, 12, e1336. https://doi.org/10.1371/journal.pone.0001336

Gold, B.J., Pomplun, M., Rice, N.J., \& Sekuler, R. (2008). A new way to quantify the fidelity of imitation: Preliminary results with gesture sequences. Experimental Brain Research, 187, 139-152. https://doi. org/10.1007/s00221-008-1291-2

Goldenberg, G. (1996). Defective imitation of gestures in patients with damage in the left or right hemispheres. Journal of Neurology, Neurosurgery, and Psychiatry, 61(2), 176-180.

Goldenberg, G., \& Hagmann, S. (1997). The meaning of meaningless gestures: A study of visuo-imitative apraxia. Neuropsychologia, 35(3), 333-341.

Hamilton, A.F. de C. (2014). Cognitive underpinnings of social interaction. Quarterly Journal of Experimental Psychology, 68(3), 417432. https://doi.org/10.1080/17470218.2014.973424

Hamilton, A.F. de C. (2015). The neurocognitive mechanisms of imitation. Current Opinion in Behavioral Sciences, 3, 63-67. https://doi. org/10.1016/j.cobeha.2015.01.011

Hayes, S.J., Dutoy, C.A., Elliott, D., Gowen, E., \& Bennett, S.J. (2016). Atypical biological motion kinematics are represented by complementary lower-level and top-down processes during imitation learning. Acta Psychologia, 163, 10-16. https://doi.org/10.1016/j.actpsy. 2015.10.005

Hermsdörfer, J., Mai, N., Spatt, J., Marquardt, C., Veltkamp, R., \& Goldenberg, G. (1996). Kinematic analysis of movement imitation in apraxia. Brain, 119, 1575-1586.

JASP Team (2018). JASP (Version 0.8.6)[Computer software].

Krüger, B., Bischoff, M., Blecker, C., Langhanns, C., Kindermann, S., Sauerbier, I., Reiser, M., Stark, R., Munzert, J., \& Pilgramm, S. (2014). Parietal and premotor cortices: Activation reflects imitation accuracy during observation, delayed imitation and concurrent imitation. NeuroImage, 100, 39-50. https://doi.org/10.1016/j. neuroimage.2014.05.074

Mengotti, P., Corradi-Dell'Acqua, C., Negri, G.A.L., Ukmar, M., Pesavento, V., \& Rumiati, R.I. (2013). Selective imitation impairments differentially interact with language processing. Brain, 136, 2602-2618. https://doi.org/10.1093/brain/awt194

Pan, X., \& Hamilton, A.F. de C. (2015). Automatic imitation in a rich social context with virtual characters. Frontiers in Psychology, 6(790). https://doi.org/10.3389/fpsyg.2015.00790

Petreska, B., Adriani, M., Blanke, O., \& Billard, A.G. (2007). Apraxia: A review. Progress in Brain Research, 164, 61-83. https://doi.org/10. 1016/S0079-6123(07)64004-7

Press, C. \& Heyes, C. (2008). Stimulus-driven selection of routes to imitation. Experimental Brain Research, 188, 147-152. https://doi. org/10.1007/s00221-008-1422-9

Raftery, A.E. (1995). Bayesian model selection in social research. In P. V. Marsden (Ed.), Sociological methodology 1995 (pp. 111-196). Cambridge, MA: Blackwell.

Reader, A. T., \& Holmes, N. P. (2015). Video stimuli reduce objectdirected imitation accuracy: A novel two-person motion-tracking approach. Frontiers in Psychology, 6(644). https://doi.org/10.3389/ fpsyg.2015.00644

Reader, A.T., Royce, B.P., Marsh, J.E., Chivers, K., \& Holmes, N.P. (2018). Repetitive transcranial magnetic stimulation reveals a role for the left inferior parietal lobule in matching observed kinematics 
during imitation. European Journal of Neuroscience, 47(8), 918 928. https://doi.org/10.1111/ejn.13886

Rumiati, R.I., Carmo, J.C., Corradi-Dell'Acqua, C. (2009). Neuropsychological perspectives on the mechanisms of imitation. Philosophical Transactions of the Royal Society B, 364, 2337-2347. https://doi.org/10.1098/rstb.2009.0063

Rumiati, R.I., \& Tessari, A. (2002). Imitation of novel and well-known actions: The role of short-term memory. Experimental Brain Research, 142, 425-433. https://doi.org/10.1007/s00221-0010956-x

Sacheli, L. M., Candidi, M., Era, V., \& Aglioti, S. M. (2015). Causative role of left aIPS in coding shared goals during human-avatar complementary joint actions. Nature Communications, 6(7544). https:// doi.org/10.1038/ncomms8544

Sacheli, L.M., Candidi, M., Pavone, E.F., Tidoni, E., \& Aglioti, S.M. (2012). And yet they act together: Interpersonal perception modulates visuo-motor interference and mutual adjustments during a joint-grasping task. PLoS ONE, 7(11), e50223. https://doi.org/10. 1371/journal.pone.0050223

Sacheli, L. M., Christensen, A., Giese, M. A., Taubert, N., Pavone, E. F., Aglioti, S. M., \& Candidi, M. (2015). Prejudiced interactions: implicit racial bias reduces predictive simulation during joint action with an out-group avatar. Scientific Reports, 5(8507). https://doi. org/10.1038/srep08507

Sacheli, L.M., Tidoni, E., Pavone, E.F., Aglioti, S.M., \& Candidi, M. (2013). Kinematics fingerprints of leader and follower role-taking during cooperative joint actions. Experimental Brain Research, 226, 473-486. https://doi.org/10.1007/s00221-013-3459-7
Tessari, A., Bosanac, D., \& Rumiati, R.I. (2006). Effect of learning on imitation of new actions: Implications for a memory model. Experimental Brain Research, 173, 507-513. https://doi.org/10. 1007/s00221-006-0395-9

Tessari, A., Canessa, N., Ukmar, M., \& Rumiati, R.I. (2007). Neuropsychological evidence for a strategic control of multiple routes in imitation. Brain, 130(4), 1111-1126. https://doi.org/10. 1093/brain/awm003

Tessari, A., Cretella, L., \& Rumiati, R.I. (2009). Route switching in imitation: Should I stay or should I go? in Proceedings of the 31st Annual Conference of the Cognitive Science Society (pp. 24752480). Amsterdam: Cognitive Science Society, Inc.

Tessari, A., \& Cubelli, R. (2014). Route selection in action imitation: A matter of strategic choice? Cortex, 57, 277-278. https://doi.org/10. 1016/j.cortex.2014.01.014

Tessari, A., \& Rumiati, R.I. (2004). The strategic control of multiple routes in imitation of actions. Journal of Experimental Psychology: Human Perception and Performance, 30(6), 11071116. https://doi.org/10.1037/0096-1523.30.6.1107

Wild, K.S., Poliakoff, E., Jerrison, A., \& Gowen, E. (2010). The influence of goals on movement kinematics during imitation. Experimental Brain Research, 204, 353-360. https://doi.org/10.1007/s00221009-2034-8

Williams, J.H.G., Casey, J.M., Braadbaart, L., Culmer, P.R., \& MonWilliams, M. (2013). Kinematic measures of imitation fidelity in primary school children. Journal of Cognition and Development, 15, 345-362. https://doi.org/10.1080/15248372.2013.771265 